\title{
DIFFERENTIABLE CLASSIFICATION OF SOME TOPOLOGICALIY LINEAR ACTIONS
}

\author{
BY RONNIE LEE ${ }^{1}$
}

Communicated by William Browder, August 19, 1968

Let $G$ act smoothly on $S^{n}$ with fixed point set diffeomorphic to $S^{k} 0 \leqq k \leqq n-3$, and with all other orbits of the same type and of dimension $r$. Connell, Montgomery and Yang have shown that the action of $G$ is topologically equivalent to a linear action of $S^{n}$ if $n-r \geqq 5$. The problem then arises as to what can be said about the differentiable classification of these topologically linear actions. In some special cases, the answers are known. For example, it was shown by Hirsch, Smale, and Livesay and Waldhausen that every smooth action of $Z_{2}$ on $S^{3}$ was differentiably equivalent to a linear one. Also, for high dimensional cases, the unknottedness of the codimensional 2 fixed point sphere of a smooth circle group action was shown to be true by $\mathrm{Wu}-\mathrm{Yi}$ Hsiang. However, we prove that there is a curious involution on $S^{23}$ with exactly two fixed points which is differentiably inequivalent to the linear action. Also we prove that for $i+1 \equiv 0(\bmod 4)$, and if the codimension is bigger than 2 and lower than metastable range, there are always semifree circle group actions on the standard $n$-sphere $S^{n}$ with the fixed point sets to be knotted $i$-spheres. The following are some of our main classification theorems. ${ }^{2}$

THEOREM 1. Up to orientation preserving diffeomorphisms, the set of equivalence classes of semifree actions of $S^{1}$ on $S^{2 n+2}$ with exactly two fixed points forms a group and is the same as the subgroup of weak isotopy classes of diffeomorphisms of complex projective $n$-space $C P^{n}$ which preserve the generator of $H^{2}\left(C P^{n} ; Z\right)$.

Similar classification theorems hold for semifree actions of cyclic $P$-group ( $P$, an odd prime). However, for the case of smooth involutions with exactly two fixed points it is more complicated. Let $f$ be a diffeomorphism of the real projective space $R P^{n}$ to itself, which is required to be orientation preserving if $n$ is odd, and let $Z_{2} \rightarrow S^{n}$ $\rightarrow R P^{n}$ be the universal covering over $R P^{n}$. By our assumption on $f$,

${ }^{1}$ Most of the results presented here appeared in the author's doctoral dissertation written under the direction of Professor Frank Raymond and submitted to the University of Michigan in partial fulfillment of the requirements for the $\mathrm{Ph} . \mathrm{D}$. degree.

$2 \mathrm{~W}$. Browder has obtained similar classification theorems independently. 
we may cover it by a covering map $\tilde{f}: S^{n} \rightarrow S^{n}$ which is orientation preserving. Gluing two copies of disks $D_{i}^{n+1}(i=1,2)$ together along their boundaries by $\tilde{f}$, we obtain an oriented $(n+1)$-sphere $\sum_{f}^{n+1}\left(=D_{1}^{n+1} \cup_{\tilde{f}} D_{2}^{n+1}\right)$. On each $D_{i}^{n+1}(i=1,2)$ there is a natural involution which sends $x$ to $-x$ (in the following, this action is denoted by $\left.\left(Z_{2}, D^{n+1}\right)\right)$. Since with respect to these actions $\tilde{f}$ is an equivariant diffeomorphism, there is a natural $Z_{2}$-action on $\sum_{f}^{n+1}$ with exactly two fixed points and it is denoted by $\left(Z_{2}, \sum_{f}^{n+1}\right)$.

THEOREM 2. Every smooth involution on homotopy $(n+1)$-sphere $(n \geqq 5)$ is of the form $\left(Z_{2}, \sum_{f}^{n+1}\right)$ for some $f . A$ necessary and sufficient condition for the existence of an orientation preserving, equivariant diffeomorphism of $\left(Z_{2}, \sum_{\tilde{f}}^{n+1}\right)$ to $\left(Z_{2}, \sum_{\tilde{g}}^{n+1}\right)$ is that $f$ is either weakly isotopic to $g$ or $\epsilon \circ \mathrm{g}^{-1} \circ \epsilon$ where $\epsilon$ is the diffeomorphism of $R P^{n}$ to itself defined by

$$
\epsilon\left(\left\{x_{1}, \cdots, x_{n}\right\}\right)=\left\{-x_{1}, \cdots, x_{n}\right\} \quad \text { for all }\left\{x_{1}, \cdots, x_{n}\right\} \in R P^{n} .
$$

Very close to this form are the classification theorems for semifree actions of circle group on $S^{2 n+2+i}(n>0)$ with fixed point set diffeomorphic to $S^{i}$. Since they are too complicated to summarize here, it is planned to present detailed formulation later. We will, however, indicate briefly some of the ideas involved in the proof of Theorem 2.

1. Outline of proofs. First given any smooth involution $\left(Z_{2}, \sum^{n+1}\right)$, around each of the fixed points $x_{i}(i=1,2)$ the action is linear; in other words, there are smoothly embedded equivariant disks $D_{i} \subset \sum^{n+1}$ such that the action restricted to each $D_{i}$ is the same as $\left(Z_{2}, D^{n+1}\right)$. The orbit spaces $\partial D_{i}^{n+1} / Z$ of the boundaries of $D_{i}(i=1,2)$ are the real projective spaces $R P^{n}$, and the orbit space $\left(\sum^{n+1}-\right.$ int $D_{1} \cup$ int $\left.D_{2}\right) / Z_{2}$ of $\left(Z_{2}, \sum^{n+1}-\right.$ int $D_{1} \cup$ int $\left.D_{2}\right)$ is an $h$-cobordism between them. Since $W h\left(Z_{2}\right)=0$,

$$
\left(\sum^{n+1}-\text { int } D_{1} \cup \text { int } D_{2}\right) / Z_{2}
$$

is diffeomorphic to $R P^{n} \times[0,1]$. From this, it can be shown that $\left(Z_{2}, \sum^{n+1}\right)$ is of the form $\left(Z_{2}, \sum_{f}^{n+1}\right)$ for some diffeomorphism $f$ of $R P^{n}$.

Now suppose that there is an orientation preserving equivariant diffeomorphism $F$ of $\left(Z_{2}, \sum_{\tilde{f}}^{n+1}\right)$ to $\left(Z_{2}, \sum_{\tilde{g}}^{n+1}\right)$. Let $\sum_{\tilde{f}}^{n+1}$ $=D_{1}^{n+1} \cup_{\tilde{f}} D_{2}^{n+1}$ and $\sum_{\tilde{g}}^{n+1}=D_{1}^{\prime n+1} \cup_{\tilde{g}} D_{2}^{\prime n+1}$. First consider the case that

$$
\tilde{F}\left(D_{1}^{n+1}(r)\right) \subset{D_{1}^{\prime n+1}}^{n} \text { and } \tilde{F}\left(D_{2}^{n+1}(r)\right) \subset D_{2}^{\prime n+1}
$$


where $D_{i}^{n+1}(r) \subset D_{i}^{n+1}(i=1,2)$ are subdisks of $D_{i}^{n+1}(i=1,2)$ of radii $r$. Since $\tilde{F}$ is orientation preserving, $\tilde{F} \mid D_{i}^{n+1}(r)(i=1,2)$ are orientation preserving diffeomorphisms of $D_{i}^{n+1}(r)$ into $D_{i}^{n+1}$. Now by a standard technique in differential topology, it can be shown that for some $r^{\prime}<r, \tilde{F} \mid D_{i}^{n+1}\left(r^{\prime}\right)$ are equivariantly diffeotopic to the identity map. Applying Thom's isotopy extension theorem to a suitable part of the orbit space, such diffeotopy can be extended to an equivariant, ambient isotopy of $\left(Z_{2}, \sum_{\vec{f}}^{n+1}\right)$ to $\left(Z_{2}, \sum_{\bar{g}}^{n+1}\right)$. From this, it follows that $f$ and $g$ are weakly isotopic. Suppose $\widetilde{F}\left(D_{1}(r)^{n+1}\right) \subset D_{2}^{\prime n+1}$, and $\widetilde{F}\left(D_{2}(r)^{n+1}\right) \subset D_{2}^{\prime n+1}$ then similar argument shows that $f$ is weakly isotopic to $\epsilon \circ \mathrm{g}^{-1} \circ \epsilon$.

2. Applications. (A). Browder-Livesay obstruction for desuspension. Let $\left(Z_{2}, \sum^{n+1}\right)$ be a smooth involution on the homotopy $(n+1)$ sphere $\sum^{n+1}$ with exactly two fixed points. A desuspension of $\left(Z_{2}\right.$, $\left.\sum^{n+1}\right)$ is a smoothy embedded sphere $S^{n}$ passing through the two fixed points, and invariant under the $Z_{2}$-action. On the other hand, Browder and Livesay have shown in [3] that for any two desuspensions of free involution, there is an obstruction $C\left(S_{0}, S_{1} ; T\right)$ for the concordance of these two desuspensions. It follows from our classification theorem that the obstruction for desuspension of $\left(Z_{2}, \sum^{n+1}\right)$ can be expressed in terms of this Browder-Livesay obstruction. Using the result in [1], we claim

CoRollary 3. If $n \neq 4 k+2$, and $n>6$ every involution $\left(Z_{2}, \sum^{n+1}\right)$ can be desuspended and for $n=6$, there exists an involution $\left(Z_{2}, \sum^{7}\right)$ on homotopy 7-sphere with exactly two fixed points which cannot be desuspended.

(B) Existence of exotic $Z_{2}$-actions.

Corollary 4. There is a curious involution on standard 23-sphere $S^{23}$ with exactly two fixed points which is differentiably inequivalent to the linear action.

By the result in [2] and [4], we produce an involution on a homotopy 24-sphere which is not divisible by two in $\Theta_{24}$. Desuspending such action, we obtain a smooth involution which can be shown to be differentiably inequivalent to the linear action.

(C) Knots as fixed point sets. We claim (proofs are to appear later)

THEOREM 4. For $i+1 \equiv 0(\bmod 4), 4 n+3 \leqq i$, and $n>0$ there is a semifree action of $S^{1}$ on $\left(S^{1}, S^{2 n+2+i}\right)$ whose fixed points set is a knotted $i$-sphere which bounds a framed submanifold in $S^{2 n+2+i}$ with nonzero index. 
By a connected sum operation with itself, there exists infinitely many differentiably inequivalent semifree actions of $S^{1}$ on $S^{2 n+2+i}$. If we restrict the group action to the $Z_{p}$ subgroup of $S^{\prime}$, we naturally obtain infinitely many $Z_{\boldsymbol{p}}$-actions which are mutually differentiably inequivalent.

REMARK 1. For semifree $S^{1}$ actions on $S^{8}$ with two fixed points, we are successful in showing that $\tilde{\pi}_{0}^{+}\left(\right.$Diff $C P^{3}$ ) is a subgroup of $\boldsymbol{Z}_{\mathbf{4}}$. Recent results of G. Brumfiel show that it is exactly $\boldsymbol{Z}_{\mathbf{4}}$.

Remark 2. It is not known whether the $S^{1}$-actions obtained in Theorem 4 are PL-equivalent to the linear action. In fact, it is not known to us what is the PL-classification for these kinds of smooth actions.

\section{REFERENCES}

1. I. Berstein and G. R. Livesay, Non-unique desuspension of involution, Mimeographed Notes, Cornell University, Ithaca, N. Y., 1968.

2. G. E. Bredon, $A \pi$-module structure for $\Theta_{*}$ and application to transformation groups, Ann. of Math. (2) 86 (1967), 434-448.

3. W. Browder and G. R. Livesay, Fixed point free involutions on homotopy spheres, Bull. Amer. Math. Soc. 73 (1967), 242-245.

4. M. Mahowald and M. Tangora, Some differentials in the Adams spectral sequence, Topology 6 (1967), 349-369.

University of Michigan, Ann Arbor, Michigan 48103 